Meta

Journal des traducteurs

Translators' Journal

\title{
Les anglicismes dans la presse d'information économique espagnole
}

\section{Mariette Meunier-Crespo}

Volume 32, numéro 3, septembre 1987

La fertilisation terminologique dans les langues romanes

URI : https://id.erudit.org/iderudit/002437ar

DOI : https://doi.org/10.7202/002437ar

Aller au sommaire du numéro

Éditeur(s)

Les Presses de l'Université de Montréal

ISSN

0026-0452 (imprimé)

1492-1421 (numérique)

Découvrir la revue

Citer cet article

Meunier-Crespo, M. (1987). Les anglicismes dans la presse d'information économique espagnole. Meta, 32(3), 273-277. https://doi.org/10.7202/002437ar d'utilisation que vous pouvez consulter en ligne.

https://apropos.erudit.org/fr/usagers/politique-dutilisation/ 


\section{LES ANGLICISMES DANS LA PRESSE D'INFORMATION ÉCONOMIQUE ESPAGNOLE}

MARIETTE MEUNIER-CRESPO

Université de Lyon III, Lyon, France

Le thème proposé aux étudiants de maîtrise de l'Université Jean Moulin Lyon III était, cette année : le vocabulaire de l'économie dans la presse espagnole contemporaine, étude des substantifs. Le corpus retenu était constitué par une dizaine de numéros du quotidien El País parus l'automne 1985 et dont on avait extrait la rubrique Economía y trabajo.

Au terme de cette étude, il est apparu que la dénomination "langue de l'économie ", à laquelle il est implicitement fait référence dans l'intitulé du programme, recouvre, dans notre corpus et en général, une réalité hétérogène. Les signataires des différents articles du corpus sont des journalistes, des économistes bien sûr, mais aussi des avocats, des hommes politiques, des chefs d'entreprises. Du point de vue linguistique, cette langue ne se différencie donc pas de façon significative de la langue journalistique, de celle des spécialistes des sciences humaines et de certains registres élaborés de la langue parlée. On y trouve certes un vocabulaire spécialisé dans le domaine économique, mais aussi celui de la langue commune, influencée par les tendances de l'expression médiatique. En particulier le goût pour les métaphores et pour les " locutions phraséologiques" (Bally).

À titre de comparaison avec un vocabulaire de spécialité plus homogène, nous avons étudié également les anglicismes qui apparaissent dans le Diccionario de marketing, récemment publié (1983) par la Asociación Para el Progreso de la Dirección.

Pourquoi le marketing ? Parce qu'il est admis que l'activité commerciale de nos jours recouvre la science, le savoir-faire du marketing. Le nom et la chose ont acquis une place prépondérante dans les différents discours concernant l'économie, et, en écho à ces discours, dans le langage quotidien actuel.

Quelques précisions terminologiques avant d'examiner les anglicismes de ces deux corpus : dans les substantifs, ou lexies substantivales (nous les désignerons désormais par le terme général de lexie) nous faisons, selon les analyses et la terminologie de $\mathbf{B}$. Pottier, la distinction entre lexies simples, lexies composées et lexies complexes. Par ailleurs, on pourra s'étonner de ne pas voir apparaître le terme "néologisme " dans une étude consacrée aux anglicismes. C'est que, à la suite de J. Casares (Cosas del lenguaje, Austral 1961), nous avons réservé l'appellation néologisme à ce que Casares appelle barbarismes : des créations sémantiques non nécessaires car elles concurrencent un terme " castizo " et créent une situation confuse due à une instabilité conceptuelle momentanée. L'usage se charge, par la suite, de rejeter ou de consacrer ces néologismes. Avec l'évolution constante de la langue, ils ne sont plus perçus comme des néologismes, avec la connotation péjorative que ce terme comporte, et entrent dans le lexique de la langue. Dans cette optique, factoría est un néologisme puisqu'il est en compétition avec planta. Mais oportunidad, qui élimine peu à peu ocasión, n'en est plus un, malgré les réticences 
des puristes qui persistent à souligner les différences de sens initiales entre les deux termes :

"A uno puedo ofrecérsele la ocasión de ir al polo Norte, pero puede ocurrir que tal viaje sea inoportuno" (J. Caseres). Ne quittons-nous pas le domaine de la synchronie pour celui de la diachronie avec ce genre de réticences?

En ce qui concerne les anglicismes rencontrés dans le corpus El País, nous en distinguons une première catégorie, fort voyante mais aussi fort peu nombreuse. Ce sont les emprunts, qui représentent moins de $1 \%$ du total des lexies relevées. On voit qu'ils répondent fort peu à l'image que l'on se fait habituellement des anglicismes, qui envahiraient les colonnes des journaux. Non encore intégrés par la langue, ils ne prêtent aucunement à confusion, ont un référent bien précis, et sont toujours signalés par une graphie différente.

Les intégrations phonétiques de ces emprunts (du type : boicot, lider, estándar) sont à peine plus nombreuses, et encore, ce fait est dû aux possibilités de dérivations que leur intégration dans la langue leur offre (boicotear, liderazgo, estandarizar).

Une deuxième catégorie d'anglicismes, tout aussi voyants que les précédents, est due aux phénomènes d'attraction paronymique. Il s'agit des calques sémantiques, régulièrement pourfendus par les tenants de la pureté du langage. Nous avons trouvé de belles diatribes de Fernando Lázaro Carreter, ou de Rafael Alvarado, dans $A . B$. C., et aussi dans le Diccionario de Anglicismos de Ricardo Alfaro (Biblioteca Románica Hispánica, Gredos, 1950). En fait, les calques sémantiques les plus voyants, ceux qui choquent notre perception d'une cohérence globale du vocabulaire, sont aussi les moins nombreux. Ils se réduisent à quelques néologismes, toujours les mêmes (factoría, tópico, colapso, oportunidad, balance, et quelques autres), ce qui tendrait à prouver une future intégration dans le vocabulaire courant. Par ailleurs, la plupart des calques sémantiques relevés par R. Alfero en Amérique latine n'apparaissent pas dans notre corpus d'espagnol péninsulaire.

L'attraction paronymique produit une variété plus prolifique et tout autant décriée de calques sémantiques dont l'intégration est plus avancée puisqu'ils se prêtent à la dérivation avec des suffixes hispaniques. Des lexies comme tipificación ou posicionamiento, tout en étant dérivées de mots castizos, tipo et posición, sont, par attraction paronymique, des calques sémantiques de l'anglais to tipify et to position. Les suffixes verbaux en - ficar et -ionar sont extrêmement productifs, ainsi que les suffixes substantivaux qui leur sont corrélés, en -ción et -miento. Dans son Diccionario de anglicismos, R. Alfaro donne la longue liste de ces calques admis par la Real Academia. Puisqu'il en est ainsi, on est en droit de penser que nous n'avons plus affaire à des anglicismes, et on comprend mal le courroux des puristes devant les plus récents d'entre eux qui sont déjà intégrés dans les langages de spécialité :

...presencia invasora de esos falsos derivados del vocablo castizo. Falsos porque no proceden de él, sino del inglés to position, que significa "asumir o mantener una actitud". Sobre ese verbo se han formado el posicionarse y el posicionamiento que campan por la pluma y la boca de ejecutivos y politicos. En los primeros, como todos los anglicismos que emplean, están al servicio de unas mentes y a domesticadas por cualquier ilustre "Business School " para practicar la "free enterprise economy". Se sientan en torno a la gran mesa de los grandes asuntos, atildados y bienolientes, se miran unos a otros como azores y se escuchan como ardillas, mientras debaten iniciativas y soluciones en una jerga esotérica, donde el idioma español titila tenuemente como una estrellita en la gran bandera de la Union. De pronto, expuestos y a Pros y contras, el ejecutivo más rápido tira de palabra y asesta : mi empresa se posiciona por un listing múltiple que cubre el coste operating ". A continuación, por orden. los demás se van posicionando también, etc. (Fernando Lazaro Carreter, A.B.C., 4-II-84). 
Or, posicionamiento est tout à fait intégré dans le vocabulaire du marketing: « Determinación de la posición ocupada por un producto en un mercado, considerando sus cualidades propias, las expectativas de la clientela, las imágenes de la competencia percibidas por la clientela y las posiciones y a mantenidas por la competencia " (Diccionario de marketing). Que ce terme corresponde au "positioning" anglais ou au "positionnement" français ne prouve que l'internationalisation des méthodes et des techniques, et, partant, l'enrichissement terminologique qui en est le corollaire. Les inutiles combats d'arrière-garde qui visent à préserver la " pureté " ( ?) du vocabulaire vont à l'encontre de l'usage, de l'évolution nécessaire de la langue et ressemblent souvent à des querelles de préséance. Ainsi, quoique en dise F. Lázaro Carreter à propos de posicionar :

Lo cual es lo mismo que optar, decidirse, adoptar una postura. Hay cien formulas castellanas, pero la borde se impone a la progenie de buena raza,

seule une longue locution analytique pourrait remplacer le précis posicionamiento.

Ainsi, ces calques sémantiques ne nous semblent pas des monstres néologiques, dans la mesure ou la nouveauté et la précision de leur référent s'imposent à leurs usagers, quels que soient l'histoire et l'itinéraire du mot et de la chose.

Un dernier exemple achèvera de clarifier notre position : il s'agit de desertización, jugé peu esthétique :

...desertización, acción y efecto de desertizar, verbo que no está en el diccionario y que cabria traducir como convertir lo que era fertil y cultivable en un secarral improductivo por falta de agua (R. Alvarado, A.B.C., 12-3-85).

Peut-on raisonnablement rejeter un verbe formé "como Dios manda", au profit d'interminables circonlocutions ? En revanche, dans le même article, R. Alvarado pourfend à juste titre le néologisme "Latinoamérica " qui apparaît sans nécessité aucune à côté de "Hispanoamérica". Toutefois, si l'usage répand majoritairement l'emploi de "Latinoamérica", aucune mise en garde n'empêchera le déclin de "hispanoamérica".

Nous nommerons la troisième catégorie d'anglicismes rencontrés dans notre corpus les crypto-anglicismes. Nous voilà enfin devant le gros bataillon de ce que l'on a coutume d'appeler les anglicismes et qui sont pourtant intégrés sous forme de calques morphologiques. Ces derniers, tout comme les calques sémantiques précédemment cités, relèvent de ce parallélisme des moyens d'expression des langues, disons pour simplifier, de culture occidentale. C'est par les calques que les expressions idiomatiques passent d'une langue à l'autre, témoins des échanges et de l'internationalisation des techniques. Loin de nous sembler un danger pour l'intégrité de la langue espagnole, nous pensons que c'est là le véritable outil pour une fertilisation terminologique. Les calques morphologiques, consistant à traduire la forme étrangère par son équivalent indigène, n'affectent pas, pour ce qui est du cas espagnol, la structure morphologique et syntaxique de la langue. Ces "anglicismes" ne sont plus sentis comme tels.

Les cryptoanglicismes constituent la majeure partie des lexies composées et des lexies complexes recensées dans notre corpus. Un pourcentage exact est difficile à établir dans la mesure où précisément, nous avons affaire à des lexies dont l'origine n'est plus patente. Cependant, on est en droit de supputer l'influence de l'anglais dans le domaine économico-journalistique, lorsque l'on constate le fort pourcentage d'occurrences des lexies complexes par rapport à l'ensemble des lexies substantivales : autour de $20 \%$, pourcentage appelé à augmenter avec la technicité des articles puisque dans le Diccionario de marketing, il tourne autour de $60 \%$. Les lexies complexes sont donc une nouveauté formelle de la langue actuelle, répondant à des besoins nouveaux de dénomination, pour les réalités d'une civilisation dans l'orbite anglo-américain. 
Les lexies complexes sont des groupes syntaxiques consacrés par l'usage. Elles se présentent sous les formes

- substantif + adjectif. Ex. : capital social, categoría socioprofesional, ecnomía liberal.

- substantif + syntagme prépositionnel, construit autour d'un autre substantif. Ex. : balanza de pagos, comisionista en bolsa de comercio, armonización de una gama de productos (cf. matching of a product line), jefe de ventas (cf. sales manager).

Certaines lexies complexes peuvent combiner les deux formations. Ex. : margen bruto de autofinanciación.

Il est parfois délicat de déterminer le degré de cohésion des groupes ainsi formés. La lexie complexe comporte un élément virtuel tandis que le groupe syntaxique est formé d'éléments actualisés :

precio de fábrica est une lexie complexe,

precio de la fábrica est un groupe syntaxique.

Ce critère n'est pas infaillible (fluidez de un mercado est une lexie complexe) et ne concerne que le type substantif + syntagme prépositionnel. Seule l'évolution du vocabulaire sous la pression de l'usage se charge de donner raison ou non à qui classe un groupe syntaxique dans la catégorie des lexies complexes.

Sur le total des lexies complexes recensées, les lexies du type nom + adjectif sont légèrement majoritaires. Parmi ces dernières, on note une montée des formations avec adjectif de relation, qui représentent la moitié des occurrences. Bally a montré que les adjectifs de relation ne se comportent pas comme des adjectifs qualificatifs : ils ne sont jamais antéposés, ni attributs, ils ne prennent jamais les degrés de comparaison. Des études plus précises examinent la spécificité de l'adjectif de relation (cf. syntagme prépositionnel et adjectif de relation in Cahiers de lexicologie, $\mathrm{n}^{\circ} 37,1980$ et la non-alternance entre syntagme prépositionnel et adjectif de relation, in Cahiers de lexicologie, $\mathrm{n}^{\circ} 43$, 1983).

La montée des formations avec adjectif de relation peut être imputable à une influence de l'anglais, plus riche en adjectifs que les langues romanes, et utilisant les adjectifs de relation avec une plus grande facilité (cf. Stylistique comparée du français et de l'anglais, J.P. Vinay et J. Darbelnet, Didier, 1977).

Ex. : medical students, des étudiants en médecine, estudiantes en medicina Congressional permission, l'autorisation du Congrès, la autorizacion del Congreso.

Les lexies complexes du type substantif + syntagme prépositionnel respectent quant à elles la syntaxe de l'espagnol, ainsi que les lexies composées : l'ordre des formants est toujours : déterminé + déterminant.

Les lexies composées, imputables au goût anglicisant pour les tournures synthétiques, ne représentent que 1 à $2 \%$ du total des lexies.

Ex. articulo-lider (cf. leader product)

On peut encore signaler la prédominance des composés endocentriques (casa matriz, estado miembro, traductores linguistas) et l'extrême rareté dans notre corpus des composés excentriques, du type portavoz.

En ce qui concerne notre second corpus, le Diccionario de marketing, sur un total de 4200 lexies, on relève 179 emprunts. En fait, ce chiffre se réduit à 93 (autour de $2 \%$, soit un peu plus que dans le corpus El País). Pour les 86 autres expressions anglaises, il existe une traduction espagnole à laquelle on renvoie le lecteur dans le même dictionnaire. 
Il est intéressant d'examiner ces traductions car elles ne se répartissent pas dans les différentes catégories établies plus haut, selon les mêmes proportions que les lexies du corpus El País.

Les adjectifs de relation et les lexies complexes avec adjectif en général sont moins nombreuses et laissent la majorité des occurrences aux lexies complexes avec syntagme prépositionnel.

L'ensemble des lexies du vocabulaire du marketing montre d'ailleurs une prédilection pour les lexies complexes avec syntagme prépositionnel ( $30 \%$ des occurrences). Ces dernières représentent en outre entre $55 \%$ et $60 \%$ des lexies complexes, ce qui tendrait à montrer que l'expression synthétique, imputable à l'anglais, marque le pas devant l'expression analytique. Quant aux lexies composées, tout comme dans le corpus $E l$ País, elles sont fort rares (entre 1 et $2 \%$ du total) et non significatives d'une nouvelle tendance de ce vocabulaire.

En guise de conclusion, nous dirons que la notion d'anglicisme demande à être nuancée, et surtout débarrassée de la connotation péjorative qui s'y attache dans l'esprit du public. Nous avons non seulement constaté que les néologismes n'infestent pas la langue de l'économie, mais encore que les calques, surtout lorsqu'ils respectent la structure morphologique et syntaxique de la langue emprunteuse, comme c'est le cas ici, sont la clef d'une fertilisation terminologique bien comprise. Nous aimerions terminer en citant Bally :

Le calque est appelé à donner une nouvelle impulsion aux études lexicologiques; dans 50 ans, on ne concevra plus de dictionnaire étymologique qui ne tiendra pas compte 1) de la filiation sémantique des sens, 2) de l'étude systématique des mots introduits par traduction (Traité de stylistique française, $3^{\mathrm{e}}$ éd., Klincksieck, 1951). 